\title{
Modello narrativo e semiotica nel Filocolo di Giovanni Boccaccio
}

Il Filocolo è tra le opere giovanili del Boccaccio uno dei testi piú attraversati dalla critica letteraria, sia perché in esso si manifesta per la prima volta il mito letterario dell'amore tra Giovanni Boccaccio e Maria d'Aquino, sia perché esso è, con tutta probabilità, il primo romanzo europeo e banco di prova per il Boccaccio piú maturo, per l'autore cioè del Decameron. Sull'opera, studiata assiduamente dai critici positivisti che vi leggevano ovvi riferimenti autobiografici, pesano, tuttavia, giudizi generalmente negativi per via della sua complessa strutturazione che già il Körting, nel lontano 1880, definiva come un insieme composto di "parti del tutto disparate," collegate semmai, con nessi "bizzarri" e perfino "grotteschi" (500). Il peso di questa stroncatura sembra riflettersi regolarmente anche sulla critica successiva, e perfino su lettori del Filocolo tanto penetranti, quali il Muscetta. ${ }^{1}$ Vittore Branca rivendica per primo l'unità narrativa del Filocolo e sostiene che l'opera rivela, nonostante la ricchezza e varietà degli episodi, "una solida unità psicologica" (198).

Nel presente articolo ci si propone un esame della struttura dell'opera applicando strumenti di lavoro relativamente moderni, si ricorre cioè a teorie di analisi del racconto sia di scuola formalista (Propp), che di scuola strutturalista (Greimas, Bermond) allo scopo di rilevare nella pur ricca polpa del Filocolo, un modello narrativo. Reperito tale modello, si ricorre ad esso come a elemento significante sia nell'ambito ristretto dell'opera esaminata, sia uscendo da essa per un'operazione di semiotica ad ampio raggio che inglobi i valori socio-culturali dell'autore.

Claude Cazalé-Bérard in un articolo del '71 sulle strutture narrative del primo libro del Filocolo (112), separa la narrazione riguardante il cantare, che chiama "fiction narrative," da quella che riguarda il mito letterario del Boccaccio, che chiama "fiction litteraire." Si adotta questa prima suddivisione del testo anche in questo articolo, sebbene non si accetti poi la successiva segmentazione del testo 
operata dallo studioso francese per la sua lettura del primo libro del Filocolo. ${ }^{2}$ Ognuna delle macrosequenze narrative di cui sopra, viene a sua volta suddivisa, nel presente articolo, in segmentazioni narrative minori. La finzione narrativa, comprende il paragrafo $3^{\circ}$ del primo libro fino al $97^{\circ}$ del quinto. Essa si estende a:

I. il mondo dell'aldilà.

II. il mondo storicizzato del cantare.

Si procede ora ad una segmentazione della macrosequenza definita "finzione narrativa."

\section{Analisi del mondo dell'aldilà}

Il cantare ha inizio con la descrizione dell'aldilà dominato dalla lotta tra Giove e Plutone, rappresentanti dei principî del bene e del male e come tali traducibili nel mito cristiano in Dio e Diavolo. Questa sequenza si articola in un'antefatto che vede:

a) una prima creazione (quella degli angeli) una ribellione (guidata da Lucifero)

una punizione (gli angeli dannati si trasformano in diavoli)

b) una seconda creazione (quella di Adamo e Eva); questa si articola sostanzialmente come a)

c) una terza creazione (quella di Cristo)

La terza microsequenza narrativa però si articola diversamente dalle precedenti e Dio commosso dal fatto che alla umanità manca un redentore (funzione "mancanza x") (Propp 153) vi provvede, e, fungendo da mandante, affida a Cristo il mandato di salvare l'umanità e di ristabilire un patto tra Dio e gli esseri umani. Cristo vi riesce, dando cosí una interpretazione positiva per i mandati successivi. Già con questa terza microsequenza si passa, grazie all'avvento di Cristo, dal mondo dell'aldilà al mondo della storia.

\section{Analisi del mondo storicizzato del cantare}

La situazione iniziale (i) (Propp 153), che serve a introdurre il personaggio dell'eroe o dell'eroina, si manifesta di nuovo con la funzione mancanza (x). A Lelio e a Giulia, nobili romani cristianizzati, manca infatti un erede. Santo Jacopo, con intervento miracoloso soddisfa la loro richiesta. Segue la prova difficile $\left(D^{1}\right)$ in forma di pellegrinaggio a Compustella, prova che Lelio stesso si impone. Questa 
però fallisce per intervento negativo di re Felice. Prima di morire, Lelio ha una visione che promette una riparazione futura in forma di conversione in massa. Dunque con il sacrificio di Lelio si stabilisce a sua volta un mandato futuro, quello della conversione, che lui non potrà però compiere. Giulia ha il compito di far sí che questo mandato venga trasmesso agli eredi; accolta, infatti, alla reggia, dà alla luce Biancifiore che nasce quasi contemporaneamente a Florio, figlio del re. Si conclude cosí il I $^{0}$ libro del Filocolo e anche quella parte della narrazione che è stata definita situazione iniziale. A questa fa seguito la parte narrativa detta preparatoria (Propp 153), nella quale Cupido in sembianzá di re Felice, fa nascere nei giovani Florio e Biancifiore un ardente amore.

Tanto la situazione iniziale, quanto la preparatoria, sono dominate dal "gioco della verità e dell'inganno . . . che poggia su una categoria grammaticale, quella dell'essere e del sembrare ..." (Greimas, Du sens 192). A livello tematico esso si manifesta con il fatto che Florio e Biancifiore, e prima Felice sono vittime di apparizione il cui scopo è ingannevole. Il contrasto però tra essere e sembrare è caratteristico della posizione di Biancifiore a corte. La giovane è discendente di nobile famiglia romana, ma viene in realtà considerata "popolaresca femmina" (II.7.150) dai reali di Spagna che sono pertanto liberali solo in apparenza. La posizione di Biancifiore è quindi condizionata dalla loro cognizione. Questo sapere sull'essere si esprime generalmente nel racconto attraverso delle qualificazioni o delle funzioni; nel nostro caso attraverso delle qualificazioni. Partendo dunque dalla dicotomia fondamentale tra essere e sembrare si ottiene dapprima un modello costituzionale:

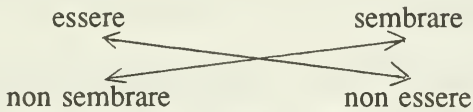

(Modello 1)

Questo modello a sua volta permette di ottenere quattro categorie d'ordine superiore: vero, menzogna, falso, segreto, ottenute dalla disgiunzione dei contrari e dalla congiunzione dei termini d'implicazione (Greimas, Du sens 145). 
vero

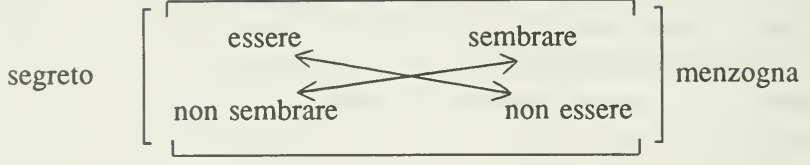

falso

(Modello 2)

Sono queste nuove categorie ottenute nel modello 2 ad articolare a loro volta la dimensione cognitiva. Biancifiore infatti, sembra pari a Florio, ma non lo è (categoria della menzogna), inoltre la posizione della giovane è caratterizzata dal segreto perché essa è ciò che non sembra (cioè è discendente di sangue imperiale, rivelazione che si effettua solo al $\mathrm{V}^{\circ}$ libro) È logico quindi che nei riguardi di Florio ci sia un divieto $\left(\mathrm{K}^{1}\right)$ implicito e di carattere sociale che lui infrange $\left(q^{1}\right)$ senza rendersene conto. Una volta che divieto e infrazione vengono esplicitati, segue l'allontanamento dell'eroe $\left(\mathrm{e}^{3}\right)$, che, a detta di Propp, comporta sempre la sciagura (n) e varie forme di danneggiamento (X).

Si passa cosí al terzo movimento, l'esordio, nel quale si verificano una serie di danneggiamenti:

I $^{\mathrm{o}}$ danneggiamento: falsa accusa di tentato avvelenamento del re per mano di Biancifiore.

II danneggiamento: alcune "giovani donzelle" cercano di far invaghire di loro Florio.

III ${ }^{\circ}$ danneggiamento: Diana suscita la gelosia di Florio contro Fileno.

IVo danneggiamento: vendita di Biancifiore ai mercanti.

La gravità di questi danneggiamenti varia nel grado di intensità e ognuno di essi è al tempo stesso una prova di difficoltà graduale. Il I $^{\mathrm{O}}$ danneggiamento mette alla prova la forza fisica di Florio (duello con Massamutino), il $\mathrm{II}^{\mathrm{O}}$ la sua costanza in amore, il $\mathrm{III}^{\mathrm{O}}$ la sua fiducia. Queste sono tutte prove qualificanti; Florio non incontra mai direttamente il vero antagonista, cioè Felice. Il IV ${ }^{\circ}$ danneggiamento infine, il piú grave, forza Florio ad abbandonare il regno per diventare eroe-cercatore. A livello tematico questo cambiamento si registra 
anche nel mutamento del nome, Florio diventa Filocolo, e intraprende un viaggio, che, a un livello, è ricerca dell'oggetto mancante, cioè Biancifiore (prova principale), e a un altro livello è, richiamandosi anche alla simbologia del viaggio dantesco, perfezionamento di sé e ricerca della verità (prova glorificante).

Da quanto detto fin qui, dunque il Filocolo sarebbe una storia di ricerca; in realtà il Filocolo è una storia con almeno due soggetti, Florio e Biancifiore, ma passando nella successiva parte del presente articolo ad un'analisi attanziale di Florio in qualità di attante del viaggio, si tralascia di parlare di Biancifiore, che nella presente analisi, figurerà unicamente nella funzione di oggetto. Oltre a ragioni di economicità pratica, questa soluzione è dovuta al fatto che Filocolo, a detta della critica, è personaggio schermo del Boccaccio, e, a detta del narratore stesso, esso è una sua proiezione. Sarà quindi un'analisi dell'attante Filocolo a permettere una possibile estrapolazione di dati significanti nei riguardi del Boccaccio e del suo mondo.

Al momento di intraprendere il viaggio, la situazione di Florio, attante-soggetto, si riassume in una serie di disgiunzioni, sia con l'Oggetto $_{1}$, cioè Biancifiore, sia con l'Oggetto ${ }_{2}$, cioè il cristianesimo, sia con l'Oggetto ${ }_{3}$, cioè il regno. Il terzo libro del Filocolo dunque, si conclude manifestando l'isolamento del soggetto che si trova in posizione di massima disgiunzione:

$$
\left(\mathrm{S}_{1} \cup \mathrm{O}_{1} \cup \mathrm{O}_{2} \cup \mathrm{O}_{3}\right)
$$

Perché avvenga una modificazione, Florio-Filocolo deve diventare pertanto l'operatore di un fare-trasformatore che convertirà appunto le relazioni disgiuntive di cui sopra, in altrettante congiunzioni. Questo avviene attraverso un processo che si svolge per stadi e che comporta le modalità del volere $(\mathrm{mv})$, del potere $(\mathrm{mp})$, del sapere (ms) e anche quella del fare (mf), piú propriamente, nel presente caso, d'un fare trasformatore $(\mathrm{Ft})$.

Primo stadio:

allontanamento (Florio a Montoro)

modalità, volere "ella Biancifiore è l'ultimo fine de' miei desii . . ." (II.13.166)

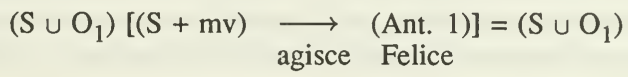


Secondo stadio:

esordio: danneggiamenti, $\mathrm{I}^{\mathrm{O}}, \mathrm{II}^{\mathrm{O}}, \mathrm{III}^{\mathrm{O}}$

modalità, potere (a livello tematico, per esempio, duello con Massamutino)

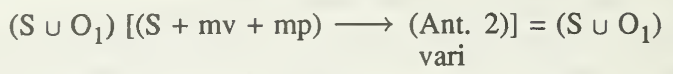

Terzo stadio:

esordio: IV ${ }^{\circ}$ danneggiamento e viaggio

modalità, sapere + fare trasformatore (la modalità del sapere si attualizza nel viaggio tramite episodi significativi, quali quello della tappa a Napoli con le "questioni amorose" e quello della tappa in Sicilia, in cui Sisife funge da informatrice).

Il risultato del fare, inteso come saper fare è confermato dal nuovo comportamento di Filocolo nell'atto di liberare Biancifiore prigioniera della torre: Filocolo, infatti non usa piú la forza (si ricordi il duello con Massamutino), ma usa l'astuzia (gioca a scacchi e si nasconde nella cesta di rose).

$$
\left(\mathrm{S} \cup \mathrm{O}_{1}\right)(\mathrm{S}+\mathrm{mv}+\mathrm{mp}+\mathrm{ms}) \longrightarrow \underset{\text { ammiraglio }}{\text { (Ant. 3) }} \Longrightarrow \mathrm{Ft}\left(\mathrm{S} \cap \mathrm{O}_{1}\right)
$$

Ad Alessandria, grazie ad una agnizione, l'ammiraglio da antagonista diventa aiutante e fa celebrare le nozze. Si verifica cosí quella modalità del far fare che d'ora in poi caratterizzerà il fare trasformatore di Filocolo. Questo fare trasformatore non è tuttavia un semplice impossessarsi dell'Oggetto ai danni di qualcuno, secondo la struttura a tre attanti: destinatario o antagonista-destinatore o soggetto-oggetto, ma è invece una capacità di fare che, acquistata dal soggetto, si trasmette ad altri e diviene quindi un far fare (come appunto nell'episodio appena illustrato). Le nozze, dunque, sono tematicamente il suggello di questa congiunzione tra soggetto e oggetto.

Il quinto libro contiene la narrazione del viaggio di ritorno. A livello tematico, l'episodio degli incontri con esseri metamorfosati è significativo in senso figurale. La modalità del far fare si attualizza negli incontri, ora positivi, tra Filocolo e Fileno, e Filocolo e Coleon, che erano personaggi vittime nel viaggio di andata. Giunto a Roma, Filocolo completa l'esperienza già iniziata nel viaggio; diventa ancora una volta operatore di un fare trasformatore che gli permetterà la congiunzione con l'Oggetto ${ }_{2}$, cioè il cristianesimo. L'analisi di 
questa sequenza ripete sostanzialmente il pattern precedentemente illustrato. La modalità del volere s'esplica, a livello tematico, quando Filocolo ammira un'immagine di Cristo; quella del potere nel trovarsi Filocolo a Roma e tra cristiani; quella del sapere infine, attraverso la predicazione di Ilario. Il cristianesimo è un valore oggetto che si acquista senza privarne altri. Il fare trasformatore ha quindi carattere squisitamente personale, cioè è un fare riflesso.

Nel Filocolo, però, anche questa esperienza viene trasmessa ad altri e il fare diventa anche in questo caso un far fare, cioè un fare causativo che transita da Filocolo agli amici prima, e in un secondo tempo a tutto il popolo di Spagna. Chiamerò pertanto questo fare transitivo o causativo, fare sociale,

$$
(\mathrm{S}+\mathrm{mv}+\mathrm{mp}+\mathrm{ms}) \underset{\mathrm{Ft}}{\Longrightarrow} \text { paganesimo }=\left(\mathrm{S} \cap \mathrm{O}_{2}\right)
$$

\section{$\mathrm{Ft}$}

Infine, l'ultima congiunzione con l'Oggetto ${ }_{3}$, il regno, si verifica ai danni di re Felice che ne viene privato. Questi però è già convertito al cristianesimo e non funge piú da antagonista. La trasmissione del regno, infatti, avviene in modo naturale, tramite la morte del re e l'eredità del titolo da parte di Florio. A ben guardare, tuttavia, questa successione segue l'iter già descritto. Essa, infatti, avviene dopo tre cerimonie funebri: la seppellizione delle ossa di Lelio, simbolo del volere, quella di Ascalion, simbolo del sapere e infine quella di re Felice, simbolo del potere (e anche del sapere e del volere). Florio, simbolo del fare trasformatore, sussume, salendo al trono, tutti questi personaggi. Finisce cosí quella vasta unità narrativa che è la "fiction narrative," si passa ora all'analisi della "fiction litteraire."

ANALISI DELLA FINZIONE LETTERARIA $\left(1^{\circ}\right.$ e $2^{\circ}$ paragrafo del $I^{\circ}$ libro, e $97^{\circ}$ del $\mathrm{V}^{\circ}$ ).

Analoga suddivisione a quella della finzione narrativa caratterizza questa macrosequenza che ingloba il testo del cantare. Essa pertanto comprende:

I. il mondo dell'aldilà

II. il mondo storicizzato del narratore.

\section{Analisi del mondo dell'aldilà}

Giunone s'avvede che a Roma manca un difensore contro la minaccia Sveva (mancanza $x$ ), tramite il papa trasmette il mandato di 
difesa della fede cristiana a Carlo d'Angiò; questi esegue il mandato positivamente. Si verifica quindi una catena di funzioni tipo: mancanza - mandato - riparazione.

\section{Analisi del mondo storicizzato del narratore}

Al motivo encomiastico degli Angiò, fa seguito la situazione iniziale (i), cioè la presentazione dell'eroe o/e dell'eroina. Vengono infatti introdotti nella narrativa la "gentilissima donna" e il narratore. Si verifica anche qui una mancanza (x) e precisamente, mancanza d'amore da parte del narratore. Mancanza però anche d'un'opera che degnamente canti gli amori fedeli di Florio e Biancifiore. La "gentilissima donna" che accusa quest'ultima mancanza, affida al narratore il compito di colmarla. Il narratore funge perciò da attante-soggetto, con doppia parte, quella di amante e quella di scrivente. La donna invece, è mandante, ma anche oggetto.

Il soggetto si trova, nella sequenza narrativa presa in esame, in relazione di disgiunzione sia con l'Oggetto ${ }_{1}$, cioè la donna, che con l'Oggetto ${ }_{2}$, cioè l'opera ancora da fare. La modalità del volere caratterizza la posizione del narratore che vuole scrivere perché amante e come tale, perché vuole piacere. La richiesta fatta dalla donna diventa perciò "commandamento" (78). L'opera diventerà "la prova per il nuovo autore," cosí si definisce il narratore (80), mentre l'amante rimarrà chiuso in una relazione disgiuntiva con l'Oggetto, cioè la donna, perché incapace di superare la modalità del volere (cioè il desiderio). Nasce da qui quella necessità di mimitizzarsi nei vari personaggi del libro e quel rapporto di metonomia con il libro stesso. ${ }^{3}$

Questa sequenza narrativa è caratterizzata dunque da una doppia disgiunzione:

$$
\left(\mathrm{S} \cup \mathrm{O}_{1} \cup \mathrm{O}_{2}\right)
$$

Alla fine dell'opera il lettore assiste solo al superamento di una delle relazioni disgiuntive, quella con l'opera. Questo avviene secondo il pattern già illustrato e il soggetto in qualità di narratore, può dare inizio all'opera perché gli è permesso dal mandante e sa scrivere l'opera. Di questa sua competenza, lo scrivente dà frequenti saggi nell'invio, dove elenca con la dovuta umiltà alcuni autori che l'hanno in-formato (modalità del sapere) Virgilio, Lucano, Stazio, soprattutto Ovidio e Dante stesso. Non ci sono infatti dubbi che il Filocolo intenda fare sfoggio di cultura letteraria e che in esso il giovane 
Boccaccio operi un notevole sincretismo di motivi e stilemi della migliore tradizione letteraria che l'ha preceduto (Muscetta 58).

Il fare trasformatore è esemplificato al massimo dall'operato di questo narratore che è in effetti un vero e proprio rifacitore, un trasformatore cioè d'un prodotto letterario già esistente. Si prenda quindi con la dovuta cautela la professione di modestia che caratterizza l'invio. In questo sdoppiarsi del soggetto tra l'insuccesso dell'amante e il successo dello scrivente, pare che solo al primo siano da attribuire le attestazioni di modestia, mentre il secondo è ben conscio di aver fatto un'opera diversa dai modelli e senz'altro piú adatta al pubblico che si è scelto. Il soggetto, infine, delega al libro stesso la modalità del far fare, cioè del fare causativo e il libro sarà 'esempio' per tanti giovani amanti. Non solo ma sarà ancora il libro, cioè l'opera compiuta a attualizzare in forma metonimica quella congiunzione con la donna che per l'amante era rimasta solo virtuale.

Si può dunque affermare che il Filocolo è strutturato su un modello narrativo che si ripete puntualmente sia nella "fiction narrative" che in quella "litteraire" e che si snoda in:

situazione iniziale: disgiunzione

situazione mediana: prova

situazione finale: congiunzione.

Visto cosí questo potrebbe per la verità essere anche lo schema della Divina Commedia. Nel Filocolo, però, le modalità dell'attualizzarsi della prova sono, anche per la loro ridondanza, significanti. L'insistenza su un modello del fare che è un fare trasformativo soprattutto transitivo, rivela infatti la distanza che corre tra l'autore della Commedia e il Boccaccio. Nel Filocolo falliscono quei soggetti che non sono operatori del fare causativo, che non operano cioè transitivamente nella società: Lelio, soggetto della sola modalità del volere nella finzione narrativa e a sua volta il narratore-amante nella finzione letteraria. Florio dichiara a un certo punto del viaggio-ricerca di non essere Enea; il narratore raccomanda al suo libro di non porsi vicino al sommo Dante. Il viaggio di Filocolo è un viaggio circolare, terrestre, circoscritto all'area del Mediterraneo; il lavoro compiuto dal nuovo narratore è anch'esso pertinente a un pubblico ben specificato, quello dei giovani e delle giovani, forse coetanei dello scrivente, certo contemporanei. La direzione dal basso verso l'alto della Divina Commedia è cambiata nel Filocolo in direzione orizzontale, cioè 
terrena. Al viaggio dantesco, simbolo d'un fare riflessivo perché conversione e salvezza dell'anima, anche se con finalità esemplari, il Boccaccio, in tempi d'un umanesimo a base già allargata, sostituisce un viaggio che è simbolo d'un fare transitivo, anzi d'un fare che si è definito sociale, proprio per quella sua dipendenza alla struttura del tempo, "ora" e dello spazio, "qui."

\section{York University}

\section{NOTE}

1 Muscetta 58. Giudizi sfavorevoli vengono anche espressi dal Marti (25). Tra gli americani lo stesso Hollander continua a parlare di "azione complicata," (39). E infine tra gli ultimissimi che si sono occupati del Filocolo, si ricorda qui Bruni che definisce anche lui la narrazione del Filocolo, "sovrabbondante, disordinata" (6).

2 Ci sembra infatti che l'autore forzi alcune sequenze narrative per ottenere delle unità strutturali omologhe da far rientrare in uno schema piú generico $\mathrm{e}$ tripartito (117).

3 Sul significato della simbologia del libro nella cultura medievale, si legga l'ottimo saggio di Picone.

\section{OPERE CONSULTATE}

BRANCA, Vittore. Boccaccio medievale. Firenze: Sansoni, 1956. Ristampato 1964.

BREMOND, Claude. Logique du récit. Paris: Edition du Seuil, 1973.

BRUNI, Francesco. "Il Filocolo e lo spazio della letteratura volgare." Miscellanea di studi in onore di Vittore Branca. Firenze: Olschki, 1983.

CAZALÉ-BERARD, Claude. "Les structures narratives dans le premier livre du Filocolo de Giovanni Boccaccio." Revue des études italiennes. N.S. 17 (1971).

GREIMAS, Algirdas J. Sémantique structurale. Paris: Larousse, 1966.

. Du sens. Essais semiotique. Paris: Edition du Seuil, 1970.

HOLLANDER, Robert. Boccaccio's Two Venuses. New York: Columbia U P, 1977.

KÖRTING, Gustav. Boccaccio's Leben und Werke. Leipzig, 1880.

MARTI, Mario (ed.). Giovanni Boccaccio. Il Filocolo. Opere minori. Milano: Rizzoli, 1969.

MUSCETTA, Carlo. Giovanni Boccaccio. Roma-Bari: Laterza, 1974.

PICONE, Michelangelo. "Tipologie culturali: da Dante a Boccaccio." Strumenti critici (giugno 1976).

PROPP, Vladimir. Morfologia della fiaba. A cura di Luigi Bravo. Torino: Einaudi, 1966. 